\title{
Smectic $A_{1}-$ Smectic $A_{d}$ Transition in Binary Mixtures of Compounds with Strongly Polar Terminal Groups
}

\author{
Ch. Bahr*, G. Heppke*, and R. Shashidhar** \\ Z. Naturforsch. 40a, $1311-1315$ (1985); received October 10, 1985 \\ On the basis of high pressure studies we present here the evidence for the existence of a smectic \\ $A_{1}$-smectic $A_{d}$ transition at high pressure in a binary liquid crystal mixture consisting of \\ terminally polar compounds. A feature of this observation is that both the constituent \\ compounds are three phenyl ring systems wherein the bridging dipoles are disposed additive \\ with respect to the polar end group.
}

\section{Introduction}

A smectic A liquid crystal can be described as an orientationally ordered fluid with a one-dimensional mass density wave along the optic axis $[1,2]$. This mass density wave can be mathematically represented by expanding the centre of mass density $\varrho(r)$ in a Fourier series. It has been pointed out by Meyer and Lubensky [3] that the symmetry of the $S_{\mathrm{A}}$ phase does not necessarily correspond to the onset of just one Fourier component and that the development of the mass density has to be periodic but may contain higher harmonics of $\varrho(r)$. The first evidence of the coupling between the fundamental and the second harmonic of the Fourier series has been obtained by Sigaud et al. [4]. While studying the phase diagram of mixtures of strongly polar 4- $n$-pentyl-phenyl-4'-cyanobenzoyloxybenzoate (DB5CN) and the non-polar compound terephthalbis-4- $n$-butyaniline (TBBA), they found evidence of a phase transition between two forms of $S_{\mathrm{A}}$ which are optically identical. Subsequent X-ray studies showed that one of the A phases was a monolayer phase $\left(\mathrm{A}_{1}\right)$ wherein the layer spacing $(d)$ is approximately equal to the length of the molecule $(l)$, while the second A phase was found to be a bilayer phase $\left(\mathrm{A}_{2}\right)$ with $d \approx 2 l$. This discovery of Sigaud et al. [4] has led to a lot of work on the smectic A phases exhibited by polar systems. At least four different

\footnotetext{
* Technische Universität Berlin, Sekr. ER 11, D-1000 Berlin 12.

** Raman Research Institute, Bangalore 560 080, India. Reprint requests to Prof. Dr. G. Heppke, Technische Universität Berlin, Sekr. ER 11, D-1000 Berlin 12.
}

forms of the A phase are known, viz., $A_{1}, A_{2}, A_{d}$ and $\tilde{A}[5,6] . A_{d}$ is a partially bilayer phase with a layer spacing intermediate between $l$ and $2 l$. $\tilde{\mathrm{A}}$ is the antiphase which has the same local order as $\mathrm{A}_{2}$ but in addition has a modulation of the dipolar heads within the layer. In addition to the $A_{1}-A_{2}$ transition observed by Sigaud et al., four other types of $\mathrm{A}-\mathrm{A}$ transitions have already been observed, viz. $A_{1}-\tilde{A}, \tilde{A}-A_{2}, A_{d}-A_{1}$ and $A_{d}-A_{2}$ $[6,7]$.

Several important features are common to all the systems which exhibit the different types of $\mathrm{A}-\mathrm{A}$ transitions discussed above:

(i) A-A transitions have so far been observed only in three-phenyl ring systems possessing a strongly polar cyano or nitro end group.

(ii) For a single component system to exhibit an A-A transition, the disposition of the bridging group dipole closest to the end chain should be such that its longitudinal component opposes that of the strongly polar end group.

(iii) In binary systems which show $\mathrm{A}-\mathrm{A}$ transitions, it has been observed that at least one constituent compound should have a bridging dipole that satisfies condition (ii).

Recently Shashidhar et al. [8, 9] reported miscibility, high pressure and X-ray studies of binary mixtures of 4-nitrophenyl-4'-(4"-hexyloxybenzoyloxy)benzoate and 4-nonyloxybenzoyloxy-4'-cyanoazobenzene. These studies showed clearly that for certain concentrations these mixtures exhibit two types of $A-A$ transitions, $A_{1}-A_{d}$ and $A_{d}-A_{d}$, the latter having been observed for the first time. It was also the only instance of an $\mathrm{A}-\mathrm{A}$ transition being observed in a binary system wherein both constituent compounds have the longitudinal components of the

0340-4811 / $85 / 1200-1311 \$ 01.30 / 0$. Please order a reprint rather than making your own copy. 
linkage dipoles oriented additively with respect to that of the end group dipole. We present here a high pressure study of binary mixtures of 4-butyloxybenzoyloxy-4'-nitroazobenzene (or 4 OBNAB) and 4-nonyloxybenzoyloxy-4'-cyanoazobenzene (or 9 OBCAB), which again shows the existence of an $A_{1}-A_{d}$ transition at high pressures.

\section{Experimental}

a) Compounds. 4OBNAB has been newly synthesized by us [10], whereas 9 OBCAB belongs to the well known nOBCAB series [11], amongst the first reentrant mesogens to be synthesized:

$$
\text { 4-butyloxybenzoyloxy-4'nit roazobenzene (4 OBNAB) }
$$
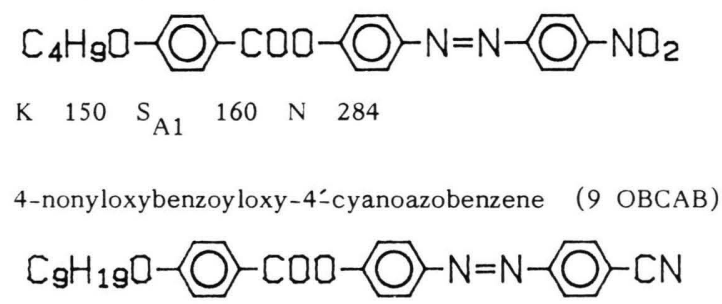

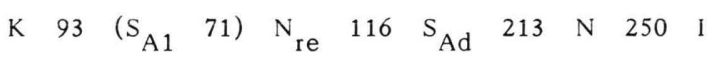

b) X-ray studies. The X-ray experiments were performed on magnetically oriented samples in sealed Lindemann glass capillaries (0.5 $\mathrm{mm}$ diameter) [12]. Copper $\mathrm{K}_{\alpha}$ radiation reflected from a bent quartz monochromator (Carl Zeiss) was used in conjunction with a flat film for recording the diffraction pattern. The temperature of the sample was maintained constant to within $\pm 0.1^{\circ} \mathrm{C}$ during any exposure, a typical exposure time being about 20 minutes. The relative accuracy of the layer spacing determination is about $\pm 0.01 \mathrm{~nm}$ or better.

c) High pressure studies. An optical high pressure cell with sapphire window was used for the experiments. This being a direct pressure transmitting cell, the sample which was sandwiched between two optically polished sapphire cylinders was isolated from the pressure transmitting medium by an elastomer material (fluran). The transition temperatures were detected by the optical transmission technique. Experiments were always conducted along isobars, i.e. the pressure was kept constant and the temperature of the sample varied at a controlled rate. The pressure was measured using a
Heise gauge to an accuracy of $\pm 1.5 \mathrm{bar}$, and the temperature to an accuracy of $\pm 0.1^{\circ} \mathrm{C}$. Further details of the pressure set up are given elsewhere $[13,14]$.

\section{Results and Discussions}

The variation of the layer spacing (d) with temperature is given in Figs. 1 and 2 for 4 OBNAB and $9 \mathrm{OBCAB}$, respectively. For $4 \mathrm{OBNAB}$ it is seen that $d$ has a constant value of $2.58 \mathrm{~nm}$ throughout the A phase. The length of the 4 OBNAB molecule measured in its most extended configuration using a Dreiding model is $2.6 \mathrm{~nm}$. Thus the A phase of this compound can be characterized as $\mathrm{A}_{1}$. The $\mathrm{A}$

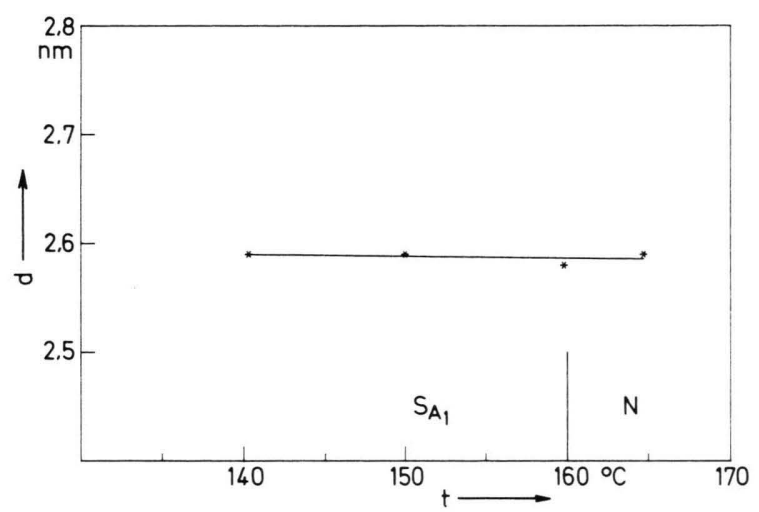

Fig. 1. Variation of the layer spacing (d) with temperature in the smectic A phase of 4 OBNAB.

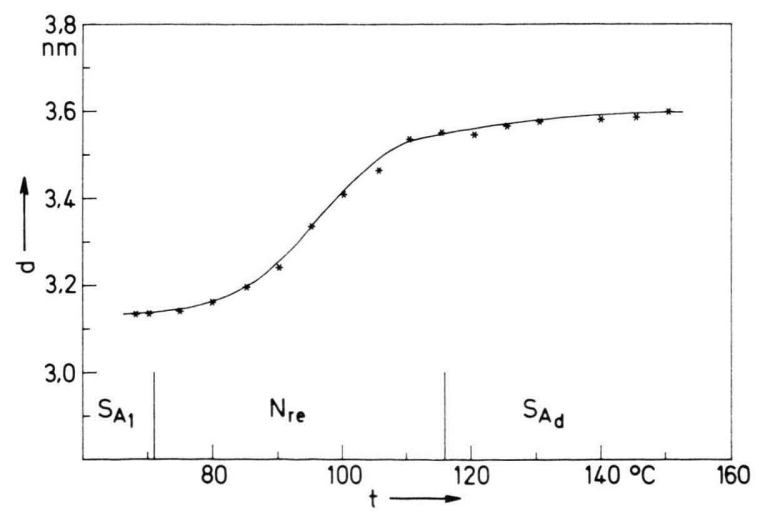

Fig. 2. Variation of the layer spacing (d) with temperature in the monolayer smectic $A\left(A_{1}\right)$, reentrant nematic $\left(N_{r e}\right)$ and partially bilayer smectic $A\left(A_{d}\right)$ phases of $90 B C A B$. 
phases in 9 OBCAB have already been established as $\mathrm{A}_{1}$ and $\mathrm{A}_{\mathrm{d}}$. The temperature variation of $d$ in this compound (Fig. 3) shows that $d$ is significantly temperature dependent in the $A_{d}$ phase while it is temperature independent in the $A_{1}$ phase [11].

The temperature-concentration diagram of binary mixtures of $4 \mathrm{OBNAB}$ and $9 \mathrm{OBCAB}$ is given in Figure 3. It is seen that addition of 4 OBNAB quickly destabilises the reentrant nematic phase which gets completely bounded in the $t-x$ plane. For $x=0.8$ ( $x$ being the mole fraction of 9 OBCAB in the mixture) there is no reentrant nematic phase and the three $A$ phases (viz., the $A_{d}$ and $A_{1}$ phases of $9 \mathrm{OBCAB}$ and the $\mathrm{A}_{1}$ phase of $4 \mathrm{OBNAB}$ ) appear to be isomorphous. It may be recalled that our earlier pressure studies on 6 ONNPBB-9 OBCAB mixtures [8] clearly demonstrated that the signature of the A-A transitions can be seen dramatically on the $\mathrm{A}-\mathrm{N}$ phase boundary in the $p-t$ plane. We have undertaken a pressure study of the $A-N$ phase transitions in $4 \mathrm{OBNAB}, 9 \mathrm{OBCAB}$ and their binary mixtures.

The $p-t$ diagram of 4 OBNAB is given in Fig. 4 while that of $9 \mathrm{OBCAB}$ is given in Figure 5. In the case of $4 \mathrm{OBNAB}$ the $\mathrm{A}_{1}-\mathrm{N}$ phase boundary is straight throughout, the phase getting bounded only because of the crystallization line intersecting the $\mathrm{A}_{1}-\mathrm{N}$ line. The $p-t$ diagram of 9 OBCAB (Fig. 5) shows the marked difference in the pressure behaviour of $A_{1}$ and $A_{d}$ phases. The $A_{d}-N$ boundary has the expected elliptic shape, characteristic of the phase diagram of reentrant mesogens. On the other hand, the $\mathrm{A}_{1}-\mathrm{N}_{\mathrm{re}}$ boundary is a straight line throughout.

It is also easy to see by extrapolation to negative pressures that the $A_{1}-N_{r e}$ and the $N_{r e}-A_{d}$ phase line would intersect at a pressure of about $-1.7 \mathrm{kbar}$ (Figure 5). This would lead to a direct $A_{d}-A_{1}$ transition at this pressure. With decrease of $x$ the pressure at which the $A_{1}-A_{d}$ transition occurs is expected to shift to higher values and for $x=0.89$ (the concentration at which the reentrant phase coexists at 1 bar) this transition would occur at atmospheric pressure. Extending the concentration to even lower values, the $x=0.80$ mixture shows that the presence of this transition manifests itself dramatically on the A-N phase boundary (Fig. 6) as an abrupt change in the slope at $0.3 \mathrm{kbar}$. An exactly similar behaviour is seen in the $p-t$ diagram of the $x=0.76$ mixture also (Figure 7). Thus,

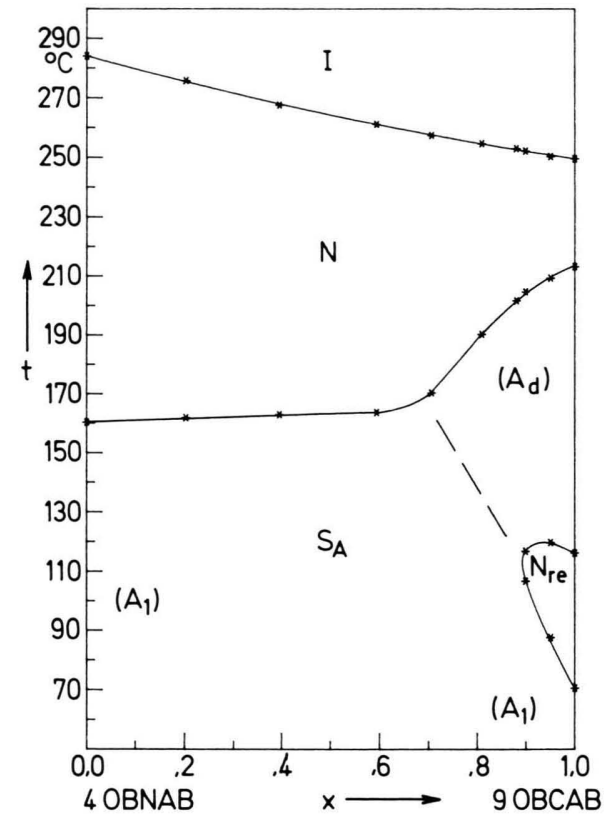

Fig. 3. Temperature-concentration diagram of binary mixtures of 4 OBNAB and 9 OBCAB. $X$ is the mole fraction of $9 \mathrm{OBCAB}$ in the mixture.

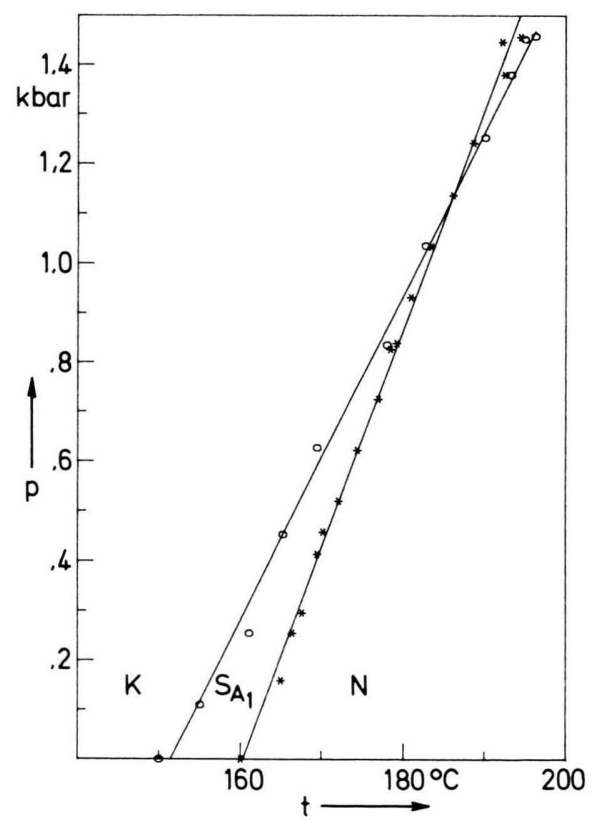

Fig. 4. Pressure-temperature diagram of $4 \mathrm{OBNAB}$. 


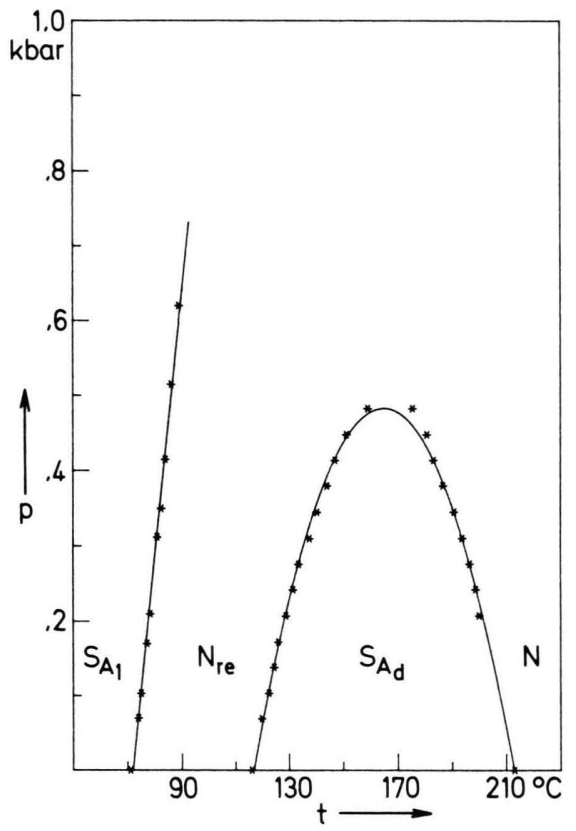

Fig. 5. Pressure-temperature diagram of $9 \mathrm{OBCAB}$.

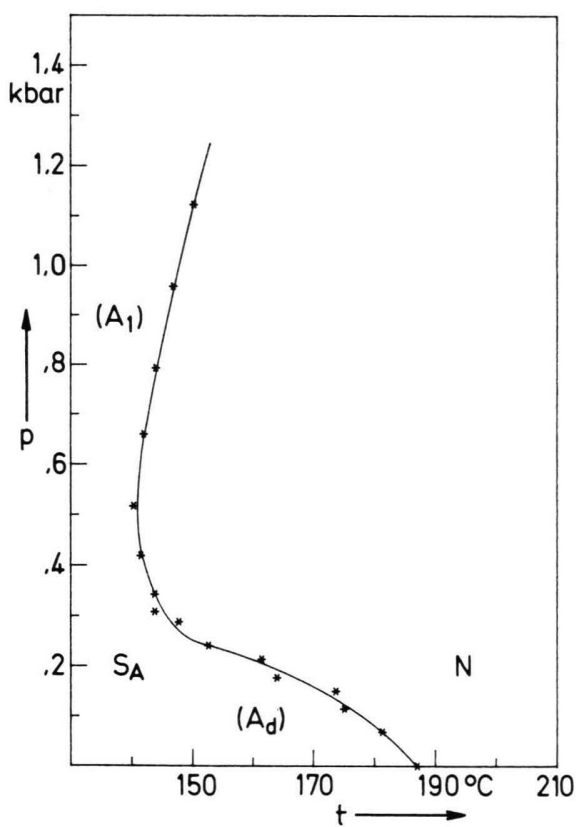

Fig. 6. Pressure-temperature diagram of the $x=0.80 \mathrm{mix}-$ ture.

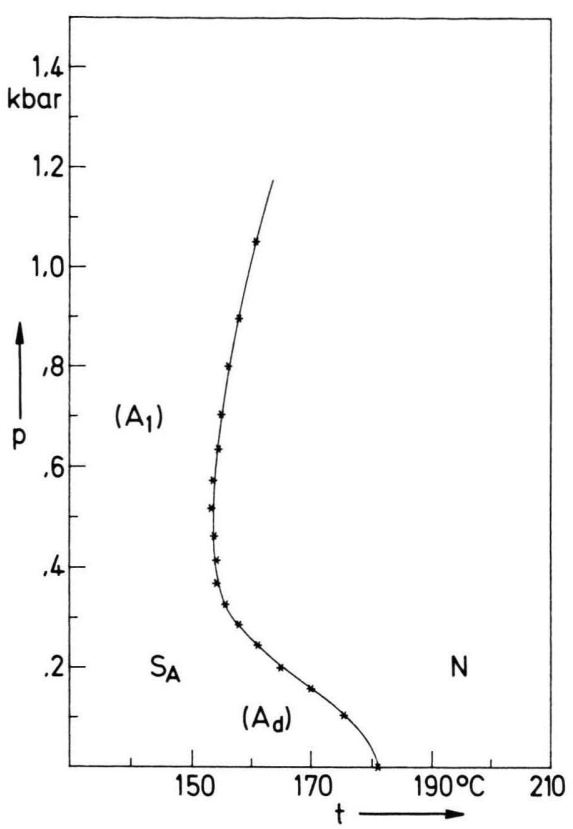

Fig. 7. Pressure-temperature diagram of the $x=0.76 \mathrm{mix}-$ ture.

although the $A_{1}-A_{d}$ transition is itself not observable at high pressure by the optical transmission method, its signature on the $\mathrm{A}-\mathrm{N}$ phase boundary is clearly seen at high pressure. It should also be pointed out that the point at which the $A_{1}-A_{d}$ line meets the $\mathrm{A}-\mathrm{N}$ phase boundary should be a bicritical point. This is because by symmetry arguments [15] the $A_{1}-A_{d}$ transition should be at least weakly first order while the $A_{1}-N$ and $A_{d}-N$ transitions are expected to be second order because of the low $T_{\mathrm{AN}} / T_{\mathrm{NI}}$ values [16]. In fact, the $p-t$ diagram for the $x=0.80$ and 0.76 mixtures resemble the topology of a phase diagram exhibiting known bicritical points [17]. Further studies are in progress to probe the $\mathrm{A}_{1}-\mathrm{A}_{\mathrm{d}}$ transition at atmospheric pressure as well as at high pressures using $\mathrm{X}$-ray diffraction experiments.

\section{Acknowledgements}

The financial support of the Gesellschaft von Freunden der Technischen Universität Berlin is acknowledged. 
[1] J. D. Litster, C. W. Garland, K. J. Lushington, and R. Schaetzing, Mol. Cryst. Liq. Cryst. 63, 145 (1981).

[2] R. Schaetzing and J. D. Litster, Advances in Liquid Crystals, Vol. 4, Ed. G. H. Brown, Academic Press, London 1979, p. 147.

[3] R. B. Meyer and T. C. Lubensky, Phys. Rev. A 14, 2307 (1976).

[4] G. Sigaud, F. Hardouin, M. F. Achard, and H. Gasparoux, J. Physique 40, C 3-356 (1979).

[5] A. M. Levelut, R. J. Tarento, F. Hardouin, M. F. Achard, and G. Sigaud, Phys. Rev. A 24, 2180 (1981).

[6] F. Hardouin, A. M. Levelut, M. F. Achard, and G. Sigaud, J. Chim. Phys. 80, 53 (1983).

[7] Nguyen Huu Tinh, J. Chim. Phys. 80, 83 (1983).

[8] R. Shashidhar, B. R. Ratna, and S. Krishna Prasad, Mol. Cryst. Liq. Cryst. Lett. 102, 105 (1984).

[9] R. Shashidhar, B. R. Ratna, and S. Krishna Prasad, Presented at the Tenth Int. Liq. Cryst. Conf., York, July 1984; Mol. Cryst. Liq. Cryst. (in press).
[10] Ch. Bahr, Diplomarbeit, Technische Universität Berlin, 1984.

[11] R. Hopf, Ph.D. Thesis, Technische Universität Berlin, 1983.

[12] B. R. Ratna, S. Krishna Prasad, R. Shashidhar, G. Heppke, and S. Pfeiffer, Mol. Cryst. Liq. Cryst. 124, 21 (1985).

[13] A. N. Kalkura, R. Shashidhar, and M. Subramanya Raj Urs, J. Physique 44, 51 (1983).

[14] A. N. Kalkura, High Pressure Optical Studies of Liquid Crystals, Ph.D. Thesis, University of Mysore, 1982.

[15] T. C. Lubensky, Private Communication.

[16] W. L. McMillan, Phys. Rev. A 4, 1238 (1971).

[17] J. Prost, Adv. Physics 33, 1 (1984). 